\title{
Pixel Value Graphical Password Scheme: Fake Passpix Attempt on Hexadecimal Password Style
}

\author{
Mohd 'Afizi Bin Mohd Shukran, Mohd Sidek Fadhil Bin Mohd Yunus \\ Faculty of Defence Science and Technology, Universiti Pertahanan Nasional Malaysia, Kuala Lumpur, Malaysia \\ Email address: \\ afizi@upnm.edu.my (M. ‘A. B. M. Shukran), sidek.my@gmail.com (M. S. F. B. M. Yunus)
}

\section{To cite this article:}

Mohd 'Afizi Bin Mohd Shukran, Mohd Sidek Fadhil Bin Mohd Yunus. Pixel Value Graphical Password Scheme: Fake Passpix Attempt on Hexadecimal Password Style. International Journal of Information and Communication Sciences. Vol. 3, No. 3, 2018, pp. 104-109. doi: $10.11648 /$ j.ijics.20180303.14

Received: November 27, 2018; Accepted: December 5, 2018; Published: December 6, 2018

\begin{abstract}
Early design of graphical password scheme solved the strong alphanumeric password deficiencies in term of hard to memorized, but they was exposed to password shoulder surfing and complicated to produced even by the legitimate users. Motivated to solve the problems, pixel value graphical password scheme was developed in 2012 where it require user to load any their desired digital image as their password. The term PassPix is referring to the pixel value that extracted from the loaded image file derived from the words password pixel. Pixel value in the form of RGB color strength could be representing as 3-octets 8-bits color code (0 to 255$)$ or Hexadecimal code ( 000000 to FFFFFF) produced $16,777,216$ combinations. Pixel value graphical password increases the password strength by implementing two-dimensional 8 by 8 grid extraction that increases the password strength to $1,073,741,824$ combinations. It would result the password could be long or super long. Currently, pixel value graphical password scheme using the 8-bits color code as the pixel value and password that stored into database which could produce up to 576 characters. This would be require more storage capacity to store the password that brings into a suggestion to extracting the pixel value using hexadecimal code which produced 384 characters only. Further study on that idea it is found that the number of password characters did not brings a significant impact on-disk data size and it is easy information to construct a fake PassPix which is discussed further in this paper. This paper is organized into 5 sections where the background and concept of pixel value graphical password scheme is introduced in section 1. Section 2 of this paper is describing the password style of pixel value graphical password. The fake PassPix reconstruction is briefly explained in section 3 and section 4 is discussed further about the fake PassPix findings. The conclusion of this study can be found in section 5 and at the end of this paper, there are list of several references used in this study. Hopefully this paper will brings major contribution for safer pixel value graphical password scheme.
\end{abstract}

Keywords: Pixel Value, Graphical Password, PassPix, RGB Color Scheme, 8-Bits Color Code, Hexadecimal Color Code

\section{Introduction}

In 2012, pixel value graphical password scheme [1] was introduce to solve the previously developed graphical password complexity and password vulnerability. Before its development, most of the graphical password scheme was involving click on image password such as Blonder's method [2] and PassPoint method [3]. By the end of 2010, click-based method was implemented widely as the additional log in security media [4]. There are also a few developments on draw-based graphical password such as signature drawing [5] and PassGo method [6]. Some of the developed graphical password scheme is complex as a method by Sobrado \&
Birget [7] and some easy to duplicate [8]. Pixel value graphical password scheme was introduced to reduce the user complexity and eliminate the password duplication issue.

With pixel value graphical password scheme, users only need to pick any of their personal desired images and load it to authentication system as their PassPix, the term for password pixel. It is far simpler for users to create and authenticate their password using this method [9] compares to the other graphical password scheme where user is freely to pick a password according to their personal preferences and they loved to memorizing it. To create a password or log in to pixel value graphical authentication system, users are requiring providing their username as their unique ID and loading their 
desired image as PassPix. The authentication interface is illustrated in figure 1.

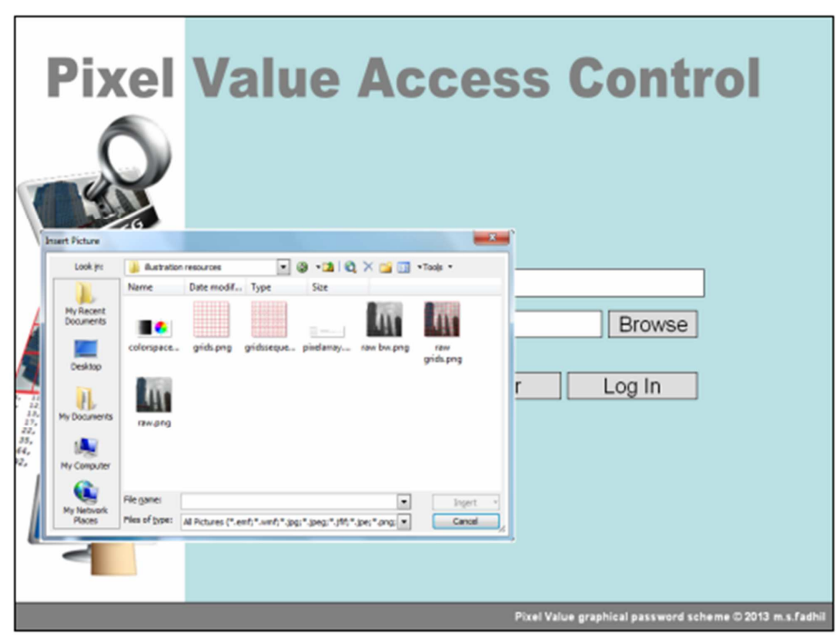

Figure 1. Pixel Value access control interface.

By allowing users to pick their image freely as their desired, users would be easily memorizing it password easily. Unlike the other graphical password, the loaded image by users are not disclosed to the other user which the password information or clue is safe. In addition, when users choose an image from their personal storage media, the PassPix is only exclusively accessible by the legitimate user.

\section{The Password Style}

For, pixel value graphical password scheme, PassPix in the form of digital image file, is the main component that carries the password information. The password will be extracted by pixel value authentication system to produce the image pixel value. The loaded image file is divided into logical two-dimensional grids and pixel value from each grids being extracted. Example of pixel value in 8-bits code and hexadecimal code $1 \mathrm{~s}$ illustrate in following table 1 .

Table 1. The 8 bits and hexadecimal pixel value color codes.

\begin{tabular}{|c|c|c|c|}
\hline Color name & Color & 8 Bits Code & Hexadecimal \\
\hline Black & & 000 & FFFFFF \\
\hline Red & & 25500 & 02550 \\
\hline Green & & 02550 & 00FF00 \\
\hline Blue & & 00255 & $0000 \mathrm{FF}$ \\
\hline White & & 255255255 & 000000 \\
\hline
\end{tabular}

Whole pixel value is arranged as one single text to be stored into the user database. The pixel value is in the form of three octets RGB color codes which could be the 8 bits color code or hexadecimal code [10]. It is a super long password and meaningless which make it a strong password. Example of an extracted 8 bits color code pixel value from an image using 8 by 8 grids is shown in figure 2 .

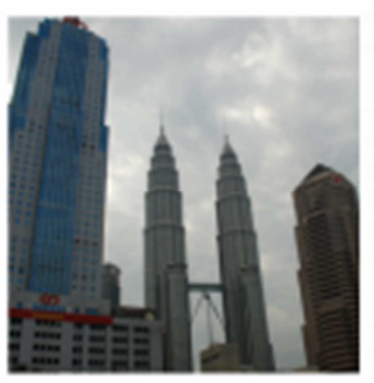

$235691,406381,6698113,839194,869089,879194,799298,305681$. $778492,808588,536875,4782110,275370,316688,286584,437290$. $234056,336898,708493,266180,325468,497087,347198,386179$. $9195106,718896,476577,868789,838892,778691,627583,416376$, $191193192,194196195,194198197,210212209,210212211,215215213,239236227,224225219$. 196198197, 186190191, 197199198, 211213210, 207209208, 210212209, 221222216, 205207202. $188192193,189193194,203205204,196198197,798887,858990,485658,647070$. $187191192,190192191,189193194,183187188,188192191,191193192,196198195,192194191$.

Figure 2. 8-bits color code password output.

Output password:

2356914063816698113839194869089879194799298305681778492808588536875478211027537031668828658443729023405633689870849326618032546 8497087347198386179919510671889647657786878983889277869162758341637619119319219419619519419819721021220921021221121521521323923 6227224225219196198197186190191197199198211213210207209208210212209221222216205207202188192193189193194203205204196198197798887 858990485658647070187191192190192191189193194183187188188192191191193192196198195192194191

When the password is arranged into a single text, it would form a 192 characters password and up to 576 characters password. The non-fixed and mash number of characters in 8-bits color code, from 000 to 255255255 , would result the number of grids and the color code combination is hard to identify for further fake PassPix reconstruction. However, when the pixel value is extracted in the form of hexadecimal codes, it is otherwise where the hexadecimal code is always fixed 6 characters.

\section{Fake PassPix Reconstruction}

Even though both hexadecimal and 8 bits color code produced 1,073,741,824 password combination using 64 grids ( 8 by 8 grids), the hexadecimal text structure is fix to 6 characters. Unlike the 8 bits color code, minimum character for each grid is 3 character and up to 9 character which it is perfect for confusing the color combination. Example of the hexadecimal password output is shown in figure 3 . 


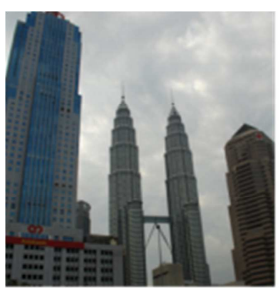

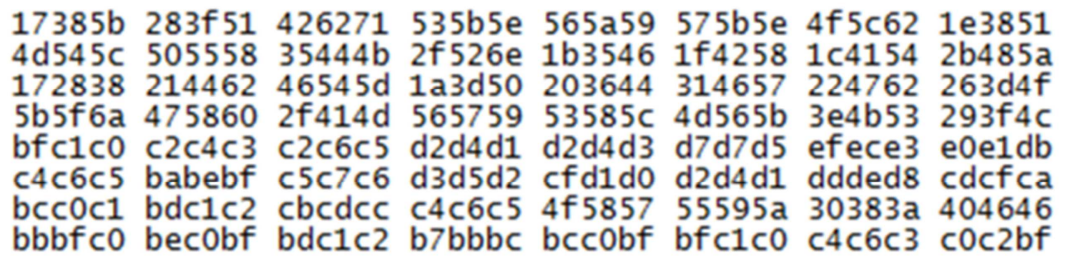

Figure 3. The Hexadecimal color code password output.

Output password:

17385b283f51426271535b5e565a59575b5e4f5c621e38514d545c50555835444b2f526e1b35461f42581c41542b485a17283821446246545d1a3d502036443146 $57224762263 \mathrm{~d} 4 \mathrm{f} 5 \mathrm{~b} 5 \mathrm{f} 6 \mathrm{a} 4758602 \mathrm{f} 414 \mathrm{~d} 56575953585 \mathrm{c} 4 \mathrm{~d} 565 \mathrm{~b} 3 \mathrm{e} 4 \mathrm{~b} 53293 \mathrm{f} 4 \mathrm{cbfc} 1 \mathrm{c} 0 \mathrm{c} 2 \mathrm{c} 4 \mathrm{c} 3 \mathrm{c} 2 \mathrm{c} 6 \mathrm{c} 5 \mathrm{~d} 2 \mathrm{~d} 4 \mathrm{~d} 1 \mathrm{~d} 2 \mathrm{~d} 4 \mathrm{~d} 3 \mathrm{~d} 7 \mathrm{~d} 7 \mathrm{~d} 5 \mathrm{efece} 3 \mathrm{e} 0 \mathrm{e} 1 \mathrm{dbc} 4 \mathrm{c} 6 \mathrm{c} 5 \mathrm{babebfc} 5 \mathrm{c} 7 \mathrm{c} 6 \mathrm{~d} 3 \mathrm{~d} 5$ d2cfd1d0d2d4d1ddded8cdcfcabcc0c1bdc1c2cbcdccc4c6c54f585755595a30383a404646bbbfc0bec0bfbdc1c2b7bbbcbcc0bfbfc1c0c4c6c3c0c2bf

Not just it produced shorter password space, the code is guessable. Since hexadecimal code is always 6 characters, the information about number of grids and the pixel value for each grids is exposed. Assuming there is no information about the grid dimension used, by counting 6 characters to form a block from that super long password, the total number of grids applied for this password can be found. To form a two-dimensional grid, simply just square root the total number of counted blocks and arrange the blocks accordingly.

For an example:

Output Password:

$17385 \mathrm{~b} 283 \mathrm{f} 51426271535 \mathrm{~b} 5 \mathrm{e} 565 \mathrm{a} 59 \ldots \ldots \alpha$

Divide text into block of 6 characters:

\section{$17385 b \quad 283 f 51 \quad 426271 \quad 535 b 5 e \quad 565 a 59 \quad \ldots \ldots \alpha$} Count the number of blocks: [1] [2] [3] [4] $[5]$ $17385 b \quad 283 f 51 \quad 426271 \quad 535 b 5$ e $\quad 565 a 59$

Calculate the dimension:

$\sqrt{ } n=Y \times x$

(example based on figure 3: 64 blocks, $\sqrt{64}=8 \times 8$ dimension)

Where;

$$
\begin{aligned}
& \alpha=\text { last character } \\
& n=\text { sum of blocks } \\
& Y=\text { size of } Y \text { axis } \\
& \varkappa=\text { size of } X \text { axis }
\end{aligned}
$$

\section{$\mathrm{X}$ axis $=8$ Blocks}

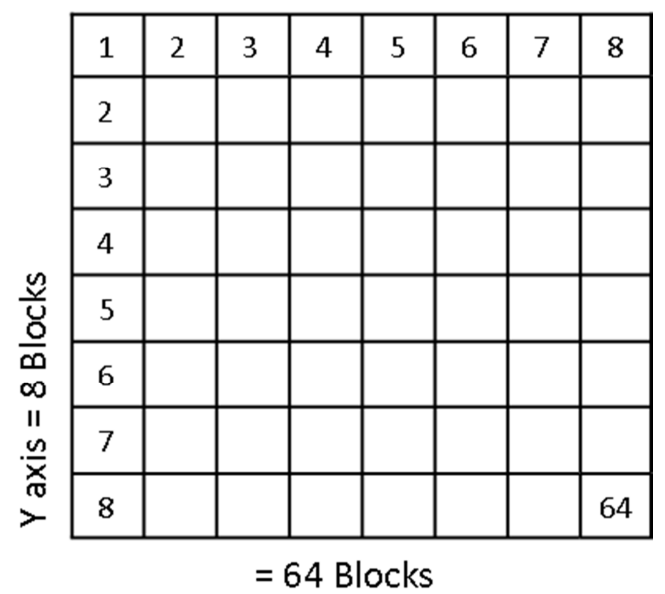

Figure 4. Two-dimensional grids reconstruction.
From the example, the total blocks count is used as the information to find the axis size where square root of 64 blocks is 8 . It is mean that both dimension axis, $\mathrm{X}$ and $\mathrm{Y}$, have the size of 8 blocks. When the size of $\mathrm{X}$ axis and $\mathrm{Y}$ axis is known, the two-dimensional grids can be constructed as illustrated in figure 4.

The hexadecimal code from each block acquired from the division step previously is used to fill in each block with color accordingly for each block. The arranged hexadecimal code block is illustrated in figure 5 and the colored fake PassPix is shown in figure 6.

\begin{tabular}{|lllllllll}
$17385 b$ & $283 f 51$ & 426271 & $535 b 5 e$ & $565 a 59$ & $575 b 5 e$ & $4 f 5 c 62$ & $1 e 3851$ \\
$4 d 545 c$ & 505558 & $35444 b$ & $2 f 526 e$ & $1 b 3546$ & $1 f 4258$ & $1 c 4154$ & $2 b 485 a$ \\
172838 & 214462 & $46545 d$ & $1 a 3 d 50$ & 203644 & 314657 & 224762 & $263 d 4 f$ \\
$5 b 5 f 6 a$ & 475860 & $2 f 414 d$ & 565759 & $53585 c$ & $4 d 565 b$ & $3 e 4 b 53$ & $293 f 4 c$ \\
$b f c 1 c 0$ & $c 2 c 4 c 3$ & $c 2 c 6 c 5$ & $d 2 d 4 d 1$ & $d 2 d 4 d 3$ & $d 7 d 7 d 5$ & efece3 & e0e1db \\
$c 4 c 6 c 5$ & $b a b e b f$ & $c 5 c 7 c 6$ & $d 3 d 5 d 2$ & $c f d 1 d 0$ & $d 2 d 4 d 1$ & ddded8 & $c d c f c a$ \\
bcc0c1 & bdc1c2 & $c b c d c c$ & $c 4 c 6 c 5$ & $4 f 5857$ & $55595 a$ & $30383 a$ & 404646 \\
bbbfc0 & bec0bf & bdc1c2 & b7bbbc & bcc0bf & bfc1c0 & c4c6c3 & c0c2bf
\end{tabular}

Figure 5. Hexadecimal code arrange into 64 blocks.

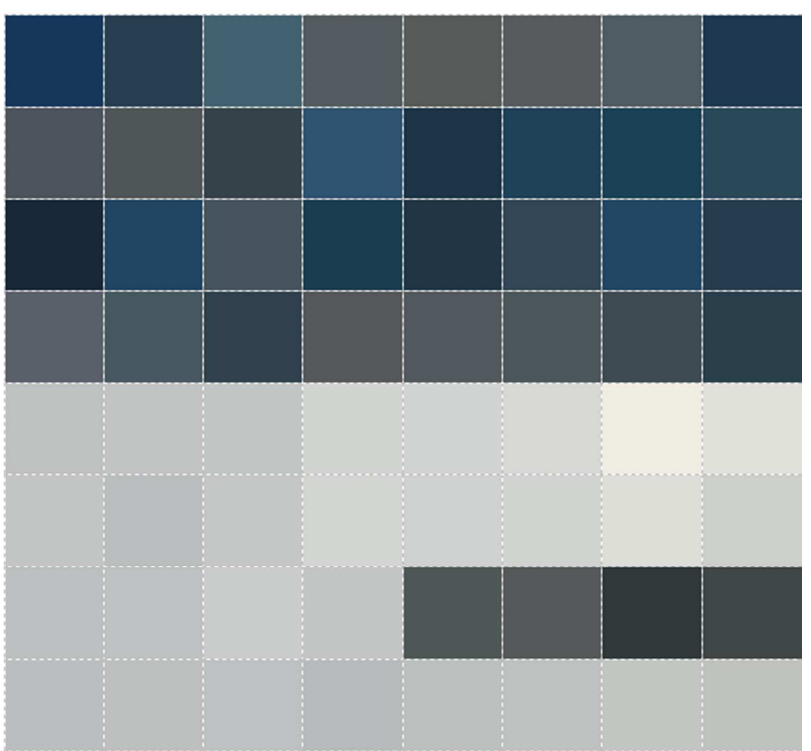

Figure 6. The grids color filling based on hexadecimal code. 
The fake PassPix is build using html table with 8 columns and 8 rows that resemblance to the two-dimensional 64 grids as illustrated in figure 4 . Every block were set 30 pixel height and 30 pixel wide with background color for each blocks as arrange in figure 6. The html file then open in browser and the colored table is capture with screen capture. The production of fake PassPix was done in the same machine where the pixel value access control system is host and the fake PassPix was tested locally. At first, the original PassPix was used to ensure that there is no system error before the fake PassPix being loaded. As expected, the PassPix was able $\log$ in as a legitimate user and $\log$ out before starting the new session.

Using the same registered user account in the database,

$\begin{array}{ll}\text { [2] } & {[3]} \\ 63,81,66 & 98,113,83 \\ 85,88,53 & 68,75,47 \\ 68,98,70 & 84,93,26 \\ 88,96,47 & 65,77,86 \\ 196,195,194 & 198,197,210 \\ 190,191,197 & 199,198,211 \\ 193,194,203 & 205,204,196 \\ 192,191,189 & 193,194,183\end{array}$

$[4]$
$91,94,86$
$82,110,27$
$61,80,32$
$87,89,83$
$212,209,210$
$213,210,207$
$198,197,79$
$187,188,188$

this fake PassPix block however, fails to gain its authentication on pixel value authentication system which discussed further next section. Before that, lets take a look on the possibility for 8-bits color code fake PassPix reconstruction.

As the 6 characters rule in a block for hexadecimal code, the 8-bits color code also has a term to be fulfilled to form a block where each block containing 3 octet numbers and each octet value are from 0 to no more than 255 . This mean, each block could containing only 3 numbers and up to 9 numbers. By using the same technique but different block rule, the password output is divided into blocks as illustrate in following figure 7 .

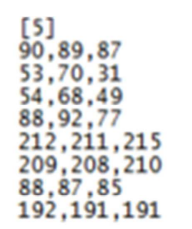

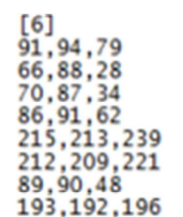
$193,192,196$

$75,83,41$
$236,227,224$
$222,216,205$
$56,58,64$
$198,195,192$

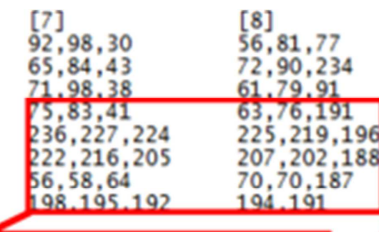

Figure 7. 8-bits code arrange into 64 blocks.

The 8-bits code was successfully arrange into 64 blocks style just like the hexadecimal code but only the last block missing the third octet. When the third octet value become unknown, a RGB color in the block 64 cannot be form and the password was incomplete. Comparison between figure 2 and figure 7 , there are few block that containing different value than the original value such as the first block and the second block that caused the last block missing its third octet where the last octet of the first block absorbing the first octet of the second block value.

This show that the key benefit of using the 8-bits code as the password text for pixel value graphical password is the method of confusing for fake PassPix reconstruction. The number of characters in each octets are not fix where it could become only one character and up to three numbers. For a block, there would be three numbers and up to nine numbers. There are also possibilities where the text becomes more confusing that would cause more octets missing or even blocks missing. The missing numbers will be remain null and could not be replace with number 0 where it produce different pixel value and the password text will be completely wrong and deny.

\section{Findings and Discussions}

A dynamic analysis on pixel value graphical password scheme in 2014 [11] conclude that, there are several factors for duplicated PassPix fail to authenticate others than the pixel value itself. Unlike the fake PassPix produced in this study, the specimen used in that testing was originally from the actual PassPix. One of the denial factors is the PassPix resolution where the pixel distribution difference will produced different pixel value. The different between the original PassPix and the fake PassPix is illustrated in figure 8 below.
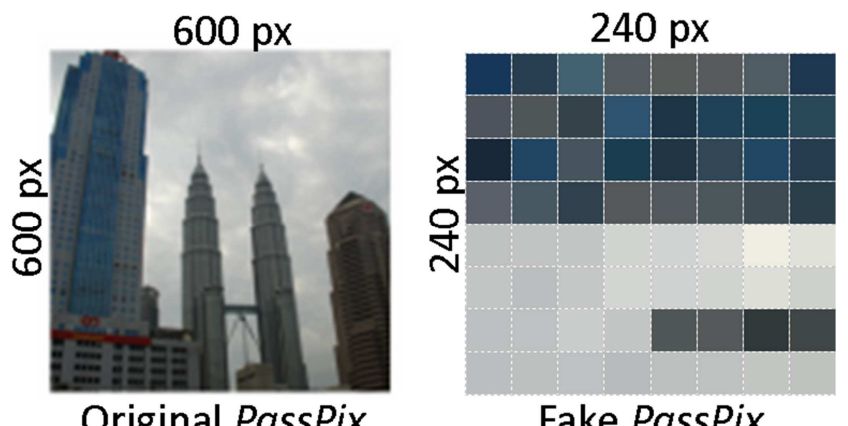

Figure 8. The difference of image resolution between original PassPix (left) and fake PassPix (right).

In the analysis study as mentioned before, the researcher explained that a small resolution digital image has gross pixel on shape edge compared to a high resolution digital image which has a sharper pixel on image edge. That makes the color on small resolution digital image scatter more on to surrounding area which affected the pixel value. In the case 
of shapeless image such as the fake PassPix, the area affected would be on the edge of each block border with the possibilities that the color on the edge is mixing among two block color and affecting the pixel value for each block.

For the second attempt, it has been decided to increase the resolution by 5 times which produce 1,200 pixels by 1,200 pixels image or 2 times larger than the original PassPix by assuming that the edge of block border are sharper than previous 240 pixels by 240 pixels fake PassPix. However, as previous fake PassPix, this large fake PassPix also failed to authenticate by pixel value access control system.

This study assumes there are two possibilities for that unsuccessful attempt. First, back to previous argument on image resolution that pixels compositions are different from the original PassPix. However, the reason for the reconstruction of larger resolution fake PassPix to get a sharper pixel edge on the border than previous fake PassPix. Then this study focus deeper on the second possibility that on the fake PassPix there are existent of several block gap, a blank area between block. It might be unnoticeable on small resolution fake PassPix and become obvious on large resolution fake PassPix. The block gap is shown on figure 9.

Even though that the table border size was set to 0 pixel and no border-color set, the block gap still exist and that make us to decide to resize that fake PassPix for 50\% less than current resolution or same as the 600 pixels by 600 pixel original PassPix. Then an image editing software is used in the operating system of the host machine. This study assumes that effort will shrink the block gap on the fake PassPix and it could become unnoticeable for pixel value access control system. However, as the previous attempt, this attempt also fails to authenticate that fake PassPix. Based on this third attempt, four assumptions have been made about the fail factor of this attempt as listed in the following table 2 .

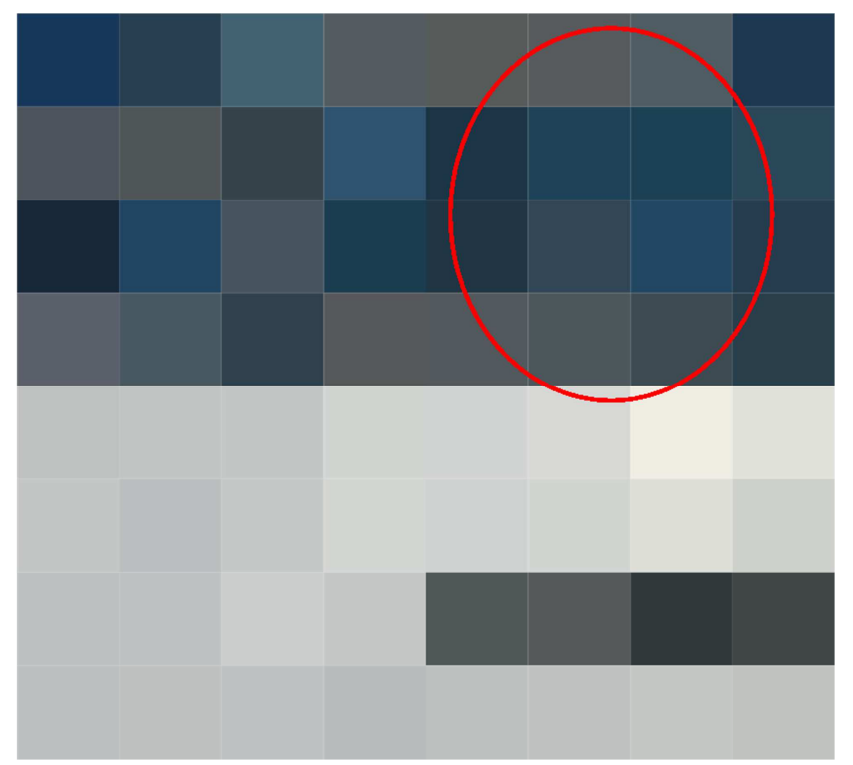

Figure 9. The block gap on fake PassPix in the rounded area and several other areas.

Table 2. Fake PassPix fail factors assumption.

\begin{tabular}{ll}
\hline Factors & Descriptions \\
\hline Block Gap & Shrunk but still exist and affected the pixel value of a block. \\
Graphic editing software colour scheme & The simple software might be fail to identify and recreate the exact colour code of each block in fake PassPix. \\
Screen capture function & As the limitation of graphic editing software, the screen capture function colour setting might be the \\
Fake PassPix resolution issue & contributing factors. \\
\hline
\end{tabular}

Ahead of this attempt, this study are not conducting further attempt due to tools limitation (such as image generator and editor software) and graphical skills. Moreover, issues on graphical tools and skills are not emphasis in this study.

As the fake PassPix did not successfully pass this attempt, it is assumed that graphical tools or software plays vital role for future attempt. For recap, the fake PassPix was generated using generic html table script and displayed on common web browser. The fake PassPix block being capture using screen capture or print screen feature and paste it on built in graphic editor application. The block on graphic editor software canvas is saved as a digital image file.

The screen capture process itself might be the cause of the fake PassPix is not successfully authenticated. The capture image needs to be crop precisely on the edge of outer border to prevent additional pixel in the fake PassPix that cause pixel value wrongly interpret. In contra, when the image being cropped smaller than the actual size, the border would not logically divide equally and cause the pixel value for blocks would be different.

Above of all, in this study, the blocks were generated using a generic html table code with 0 pixel border thickness. With common web browser as the media to display the blocks, the table border might be the cause of the block gap as shown in figure 9 before. In this case, even though the html method is the easiest way to construct the 64 blocks image, it is not a perfect method for constructing the blocks. It might be required more graphical specific tools for blocks generator.

\section{Conclusion}

This study is not promoting and encouraging the act of breaking the password especially for pixel value graphical password scheme. As the graphical password scheme researches are just 22 years old, it is require more and more research and development effort in order to make it reach the mature state as the alphanumeric password. However, the maturity of alphanumeric password does not guarantee the safety and security of the password information and those made the 6 years old pixel value graphical password scheme much safer and convenient password method to be implement. 
The password style of pixel value graphical password scheme is reside in the digital image that called as PassPix which consisted of the pixel value or RGB value of logical blocks built during the extraction process can be represent in 3 octets 8-bits codes (000 to 255255255) or hexadecimal code $(000000$ to FFFFFF). Both color code has implementation benefits where 8-bits codes is the method of confusing the pixel value blocks reconstruction and hexadecimal code has shorter text length to store into database.

Section 3 of this research has proved that the hexadecimal code is an easy target to interpret the dimension of PassPix grids and the pixel value that reside in each blocks. For 8-bits code, the text was successfully arranged into 64 blocks but the third octet of the last block is missing due to the pixel value confusion on the first block and the second block.

Even though the fake PassPix was successfully constructed based on hexadecimal code, it is still failed to bypass the pixel value access control system. The major factor of this failure is the limitation of graphical tools and skills to generate the fake PassPix digital image file. While the html table is proven not a best method, it is assumed that it will require a precise block building tools that would not producing a block gap as in figure 9 and the capability to produce the digital image file without involving the screen capture feature.

Despite of the failure of the fake PassPix method exhibited in this study, this study also have proved that it is hard and complicated effort to break the pixel value access control system. Security attempt researches such this study were highly welcome in order to make pixel value graphical password scheme is harden and trusted to become a security parameter for log in system.

\section{References}

[1] M. A. M. Shukran and M. S. F. M. Yunus, "Pixel Value Graphical Password Scheme” PI 2013003261 Malaysia, 2015

[2] G. E. Blonder, "Graphical password" 5,559,961 Washington D. C., United State of America, 2015

[3] S. Chiasson, A. Forget, R. Biddle, and P. C. van Oorschot (2009). "User interface design affects security: Patterns in click-based graphical passwords," International Journal of Information Security, vol 8, pp 387, 2015

[4] Malaysian Banking Berhad, "M2U new enhanced Security Features. Maybank 2 U," [Online] https://www.maybank2u.com.my.

[5] A. F. Syukri, E. Okamoto and M. Mambo, "A user identification system using signature written with mouse," Australasian Conference on Information Security and Privacy, vol. 13, pp. 403-414, July 2015. [Springer, Berlin, Heidelberg]

[6] H. Tao, "Pass-Go, a new graphical password scheme" Doctoral dissertation, University of Ottawa (Canada), 2017.

[7] L. Sobrado and J. C. Birget, "Graphical passwords," The Rutgers Scholar, an electronic Bulletin for undergraduate research, vol. 4, pp. 12-18, 2016.

[8] R. Biddle, S. Chiasson and P. C. Van Oorschot, "Graphical passwords: Learning from the first twelve years" ACM Computing Surveys (CSUR), vol. 44, p. 19, 2015

[9] M. A. M. Shukran, M. S. F. M. Yunus, W. S. S. Shariff, M. S. Ariffin and K. Maskat, "Pixel Value Graphical Password Scheme: Identifying Design Features and Requirements," Applied Mechanics and Materials, Vol. 548, pp. 1561-1565, 2014, Trans Tech Publications.

[10] "HTML Color Codes," [Online] https:/htmlcolorcodes.com/, September 3, 2015.

[11] M. S. F. M. Yunus, "Dynamic Analysis on Pixel Value Graphical Password Scheme,” Master dissertation, National Defense University of Malaysia, 2016. 